\title{
Molecular Mass Analysis of Antibodies by On-Line SEC-MS
}

\author{
Lowell J. Brady, John Valliere-Douglass, Theresa Martinez, and \\ Alain Balland \\ Department of Process and Analytical Sciences, Amgen, Inc, Seattle, Washington, USA
}

\begin{abstract}
Mass analysis of recombinant protein therapeutics is an important assay for product characterization. Intact mass analysis is used to provide confirmation of proper translation of the DNA sequence and to detect the presence of post-translational modifications such as amino acid processing and glycosylation. We present here a method for the rapid mass analysis of antibodies using a polyhydroxyethyl aspartamide column operated in size-exclusion mode and coupled with ESI-MS. This method allows extremely efficient desalting of proteins under acidic conditions that are optimal for subsequent mass analysis using standard ESI conditions. Furthermore, this technique is significantly faster and more sensitive than rpHPLC methods, typically considered the standard chromatography approach for mass analysis of proteins. This method is flexible and robust, and should prove useful for applications where a combination of speed and sensitivity are required. (J Am Soc Mass Spectrom 2008, 19, 502-509) (C) 2008 American Society for Mass Spectrometry
\end{abstract}

$\mathrm{M}$ ass analysis of recombinant proteins is a key characterization assay used to evaluate the entire amino acid sequence of the molecule and the presence of post-translational modifications. As part of protein drug development, intact mass analysis supports the characterization package for regulatory filings and may be used to evaluate lot-to-lot consistency on a whole molecule level. Mass analysis of intact or reduced antibodies has been used to evaluate the degree of processing of C-terminal lysine on the heavy chain subunit [1]; evaluate $\mathrm{N}$-terminal heterogeneity such as pyroglutamic acid formation [2, 3]; profile N-linked carbohydrate heterogeneity [3, 4]; and to detect instabilities in the molecule such as oxidation [5], succinimide formation from aspartic acid [6], glycation [7], internal cleavage [8], and thioether formation [9]. Reversed-phase HPLC (rpHPLC) separation followed by electrospray ionization-mass spectrometry (ESI-MS) analysis is the typical method for analyzing antibodies under both non-reduced and reducing conditions. This method is highly resolving and advantageous for the detection of minor product impurities and the resolution of different amino acid sequences or heterogeneous post-translational modifications. However, rpHPLC of antibodies has several distinct disadvantages. Due to the large size and relatively hydrophobic nature of antibodies, high temperatures are typically employed to improve elution and peak shape profiles [1,4]. However, high temperatures may lead to artifactual degradation of the sample during analysis. Inclusion of TFA

Address reprint requests to Alain Balland, Ph.D., Department of Process and Analytical Sciences, Amgen, Inc., 1201 Amgen Court West, Seattle, WA, 98119, USA. E-mail: ballanda@amgen.com as a mobile phase additive in rpHPLC is often necessary to obtain good chromatography [1], but may result in decreased ionization [10,11]. Additionally, reduction of antibodies and analysis of the constituent light and heavy chains often results in tailing of the heavy-chain component and the potential for carry-over problems. These issues can lead to lengthened run times or the inclusion of blank runs between samples to increase confidence in the results.

We optimized the use of a commercially available polyhydroxyethyl aspartamide column operated in SEC mode (pHEA-SEC) for the rapid on-line analysis of antibodies. This chromatographic material has been originally developed for the separation of polar compounds by hydrophilic interaction chromatography [12]. By operating this column in $0.1 \%$ formic acid under isocratic conditions and introducing organic solvent by post-column addition, we developed a robust and fast separation method that is highly flexible and tolerates numerous sample buffer components. Additionally, this new approach differs from other reported SEC-MS methods, achieving a rapid separation in the absence of salts [13] or organic modifiers [14]. We were able to interface this method with an ESI-TOF mass spectrometer and obtained very high quality mass data with experimental to theoretical mass agreements below $25 \mathrm{ppm}$ for several intact antibodies. Furthermore, since this method is highly tolerant of different sample buffer components, we were able to use the same method, without modification, for the analysis of reduced antibody samples. This method is a highly flexible and broadly applicable chromatography procedure for mass analysis of proteins. 


\section{Experimental}

\section{Molecules}

The CHO-expressed monoclonal antibodies (MAb) used in this study were manufactured at Amgen, Bothell, WA.

\section{Column Conditioning and Operation}

A $2.1 \times 250 \mathrm{~mm}$ polyhydroxyethyl aspartamide (pHEA) $300 \AA$ column received from the manufacturer (PolyLC, Inc., Columbia, MD) was conditioned by operating for 12 to $18 \mathrm{~h}$ at $0.2 \mathrm{~mL} / \mathrm{min}$ on $0.1 \%$ formic acid (vol/vol). After this initial conditioning step, the column was operated in the same solvent at $0.1 \mathrm{~mL} / \mathrm{min}$ at ambient temperature. The column was stored in the same solvent and samples analyzed after operating the column for 10 to $30 \mathrm{~min}$ at $0.1 \mathrm{~mL} / \mathrm{min}$. For each sample, the column was operated for $8 \mathrm{~min}$ as described in the Results and Discussion section.

\section{Post-Column Addition of Organic Solvent}

Acetonitrile with $2.0 \%$ (vol/vol) formic acid was added post-column using a stainless steel tee at $0.1 \mathrm{~mL} / \mathrm{min}$ using an LC Packings "Switchos" module (Dionex, Inc., Sunnyvale, CA). Any pump capable of providing stable flows of $0.1 \mathrm{~mL} / \mathrm{min}$ at backpressures up to 20 to 30 bar (due to constriction at the inlet of the mass spectrometer) is appropriate.

\section{Sample Preparation and Column Injection Levels}

Non-reduced samples were analyzed following dilution to $2 \mathrm{mg} / \mathrm{mL}$ with water. For reduced samples, the protein was diluted to $2 \mathrm{mg} / \mathrm{mL}$ with $20 \mathrm{mM}$ DTT in 6.0 $\mathrm{M}$ guanidine $\mathrm{HCl} / 100 \mathrm{mM}$ Tris, $\mathrm{pH} 8.0$ and incubated for $30 \mathrm{~min}$ at $37^{\circ} \mathrm{C}$. For all samples, an injection of $2 \mu \mathrm{L}$ (corresponding to $4 \mu \mathrm{g}$ ) was made onto the column except as noted in the text.

\section{Mass Spectrometry}

The outlet of the mixing tee used to add organic solvent post-column was connected to the internal divert valve attached to an Agilent TOF (model 6210) mass spectrometer (Agilent, Inc., Santa Clara, CA). Key source parameters were as follows: nitrogen gas flow of $9 \mathrm{~L} / \mathrm{min}$; fragmenter voltage of $415 \mathrm{~V}$ for non-reduced samples and $250 \mathrm{~V}$ for reduced samples; capillary voltage of $5000 \mathrm{~V}$ for non-reduced samples and $4000 \mathrm{~V}$ for reduced samples; and nebulizer pressure of 60 psi. Other conditions were standard ones used with positive ESI for the Agilent TOF. The TOF was calibrated over the range 0-4000 $\mathrm{m} / \mathrm{z}$ using Agilent ESI calibration mix before analysis with an overall mass error for all points below 3 ppm.

\section{Reversed-Phase HPLC}

Separation by rpHPLC was performed similar to the method described in [1]. Briefly, a $2.1 \times 150 \mathrm{~mm}, 300 \AA$ Zorbax SB-C8 column (Agilent) was operated at 0.3 $\mathrm{mL} / \mathrm{min}$ at $75^{\circ} \mathrm{C}$. Solvent A was water $+0.12 \%$ trifluoroacetic acid (TFA) (wt/vol) and Solvent B was 10: 80:10 water:2-propanol:acetonitrile + 0.10\% TFA (wt/ vol). Samples were loaded (levels described in the text) in $10 \% \mathrm{~B}$ and eluted with a gradient to $90 \% \mathrm{~B}$ in $20 \mathrm{~min}$.

\section{Deconvolution}

Deconvolution was performed in two ways. For nonreduced samples, spectra corresponding to chromatographic peaks were exported to text files, imported into MassLynx (Waters, Inc., Milford, MA) and deconvoluted using the MaxEnt1 algorithm. For MaxEnt1 deconvolution, an appropriate peak width value was selected and the data iterated to convergence with an output resolution of $1.0 \mathrm{Da} /$ channel; other parameters were default values for the software. For reduced samples, the deconvolution program supplied by Agilent (within MassHunter Workstation) was used in "automatic" mode.

\section{Results and Discussion}

Several features of the size-based separation achieved using pHEA-SEC chromatography make it ideal as a general method for analysis of proteins by mass spectrometry. The first advantage offered by this column is the similar elution profiles obtained for proteins of different sizes and shapes, allowing it to be used as a general method without sample specific development. In pHEA-SEC, proteins are separated from salts and other buffer components under isocratic flow conditions in aqueous solvent acidified with $0.1 \%$ ( $\mathrm{vol} / \mathrm{vol}$ ) formic acid. By adding acetonitrile post-column, proteins are introduced to the source under conditions ideal for subsequent ESI analysis. This second feature of pHEA-SEC presents an advantage over typical rpHPLC methods, which usually employ TFA as an organic modifier. The negative impact of TFA on ionization of proteins in ESI is well known [10,11]. However, TFA is often employed as an additive to rpHPLC-based separations before MS analysis because of the advantages this modifier gives to elution peak shapes [1]. Although other modifiers, such as formic acid or acetic acid, may give better ionization, the chromatographic profiles obtained for proteins are often unacceptable when these counter-ions are employed. In contrast, one significant advantage of pHEA-SEC is the ability to separate protein samples in formic acid while maintaining excellent chromatographic peak shape. To demonstrate the advantages this method offers over rpHPLC based approaches, we compared chromatography and ESI-MS results obtained for an antibody sample analyzed by pHEA-SEC compared with the same sample separated using an optimized TFA-based rpHPLC method. Figure 
1 shows a comparison of the $\mathrm{A}_{214} \mathrm{~nm}$ and total ion current (TIC) traces obtained for the sample at two different sample loads: $4 \mu \mathrm{g}$ and $20 \mu \mathrm{g}$. Comparison of the UV traces for the two methods showed similar UV peak area values once differences in flow rate between the two methods were accounted for. Although we routinely analyze $4 \mu \mathrm{g}$ of protein using pHEA-SEC with good results, this level of injection was too low to obtain good data by rpHPLC. To compare TIC traces between the two methods, injection amounts typical of the two approaches were compared (20 $\mu \mathrm{g}$ for rpHPLC and $4 \mu \mathrm{g}$ for $\mathrm{pHEA}-\mathrm{SEC})$. Even though 5-fold greater protein was injected on the rpHPLC column, the intensity obtained by pHEA-SEC was still 45-fold higher.

Inspection of the $\mathrm{A}_{214} \mathrm{~nm}$ traces shown in Figure 1 for the pHEA-SEC separation shows several aspects of the method. The intact antibody eluted at about $3.5 \mathrm{~min}$, or $350 \mu \mathrm{L}$. Since the concentrations of salts in this sample are low in proportion to the protein, no salt peak is present. However, in the $20 \mu \mathrm{g}$ injection, where a greater volume was injected on the column, a negative absorbance peak can be seen at about $7.5 \mathrm{~min}$ (or 750 $\mu \mathrm{L}$ ). This peak is due to the separation of water in the sample from the antibody; the negative peak is caused by the decrease of formic acid concentration, resulting in less absorbance. This negative peak marks the "total volume" of the column at about $750 \mu \mathrm{L}$ and has been supported by the injection of pure water (data not shown).

Figure 2 shows a comparison of the raw spectra corresponding to the $4 \mu \mathrm{g}$ pHEA-SEC and $20 \mu \mathrm{g}$ rpHPLC analyses. The excellent peak shape and signal intensity obtained by pHEA-SEC is visible in both the full range spectra and the detail view of the $48+$ charge state. In contrast, the rpHPLC introduced sample showed a much lower signal to noise ratio that can be clearly seen in both views of the raw spectra. The multiple peaks present at each charge state are due to heterogeneity of the N-linked carbohydrates found on the molecule. This is clearly shown by the deconvoluted spectrum shown for each sample in Figure 3. For each spectrum, the major species are practically identical, with $\sim 162$ Da spacing between different masses due to heterogeneous terminal galactosylation on the N-linked carbohydrates of the antibody. The correspondence of the mass data with expected values was 24 ppm for major species in each dataset. Although the pattern of major species in the deconvolution was similar for each
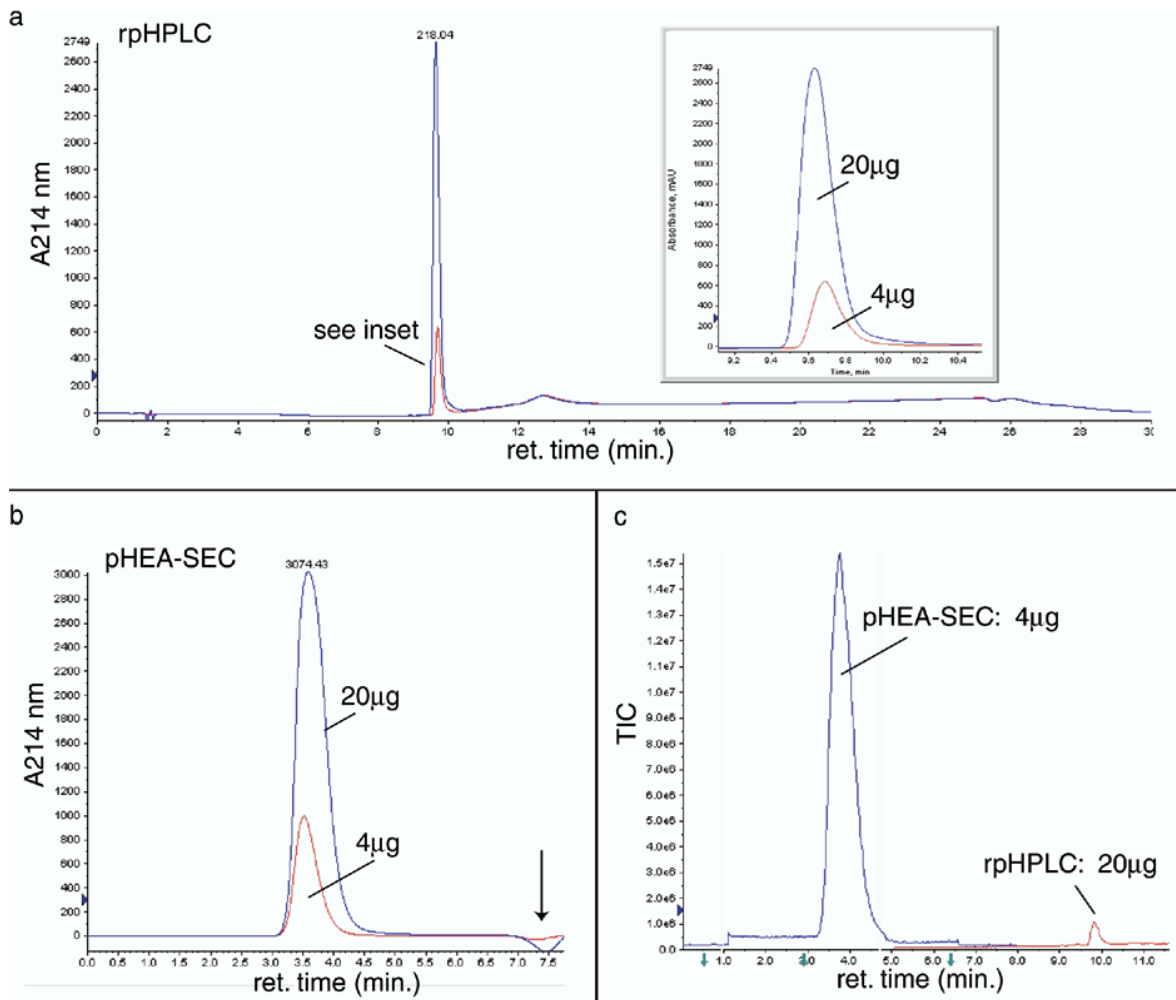

Figure 1. Comparison of the $\mathrm{A}_{214} \mathrm{~nm}$ traces obtained for a non-reduced antibody separated by rpHPLC (a) and pHEA-SEC (b) at $4 \mu \mathrm{g}$ and $20 \mu \mathrm{g}$ sample load levels. Note that the pHEA-SEC method was complete in $8 \mathrm{~min}$ while the rpHPLC runs were $30 \mathrm{~min}$ long. The arrow in (b) indicates the approximate total volume of the column as discussed in the text. For the rpHPLC analysis, only the $20 \mu \mathrm{g}$ injection yielded mass data with sufficient intensity for comparison. For the pHEA-SEC separation, both samples yielded good ionization, with the $20 \mu \mathrm{g}$ injection yielding only a 1.7 increase in MS signal over the $4 \mu \mathrm{g}$. For comparison of the total ion current (TIC) traces, typical injections were compared for each method (c). The area of the TIC trace for the $4 \mu \mathrm{g}$ pHEA-SEC run was over 45 -fold higher in intensity than that for the $20 \mu \mathrm{g}$ rpHPLC analysis. 
a
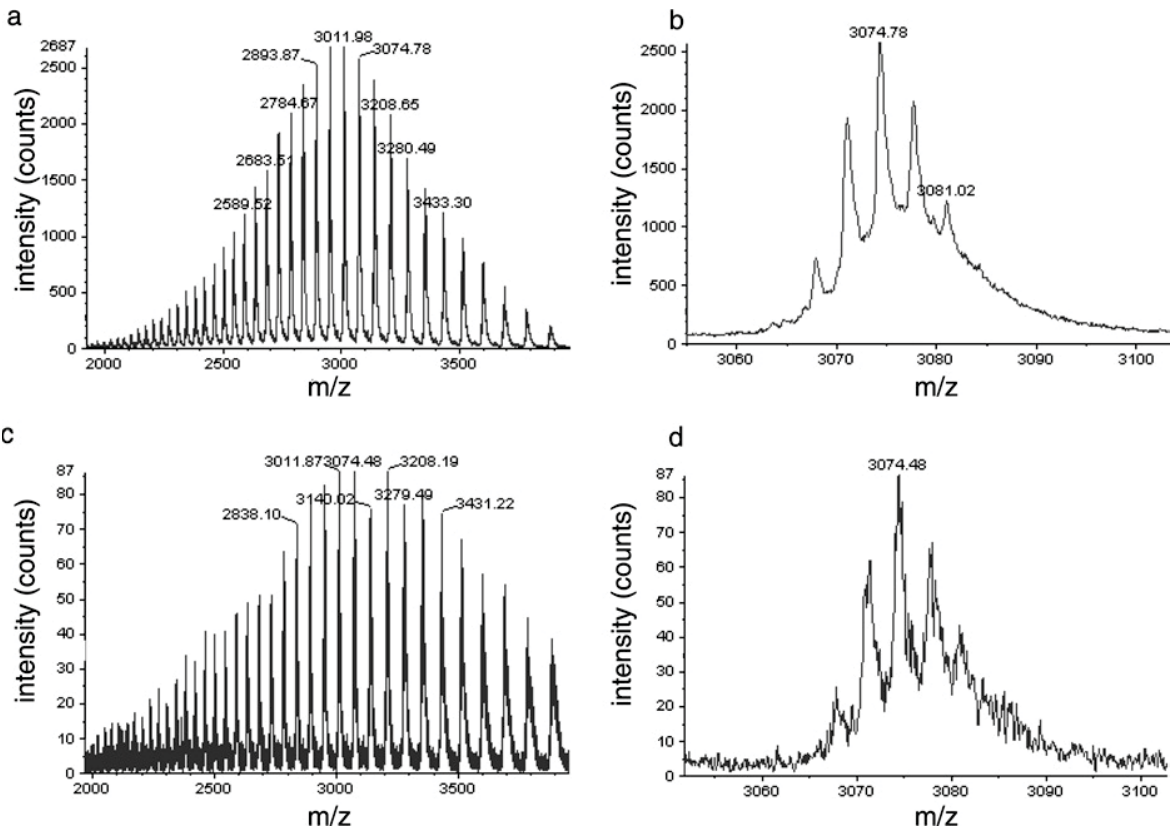

Figure 2. Comparison of the summed full-scale raw spectra obtained for both pHEA-SEC (a) and rpHPLC (c) analyses. Panels at right show a detail view of the $3074 \mathrm{~m} / \mathrm{z}$ charge-state from the pHEA-SEC (b) and rpHPLC (d) spectra. The pHEA-SEC method generates significantly higher signal and less noisy data relative to rpHPLC.

analysis, the pHEA-SEC method showed a markedly cleaner baseline, with fewer minor peaks observed. Satellite peaks present in deconvoluted data of antibodies have been previously described [15] and are a common (and not fully understood) hallmark of this type of analysis. Reduction of these artifacts in the deconvoluted data obtained by pHEA-SEC simplifies the interpretation of the data and is an advantage of the method over rpHPLC based approaches.

To show the flexibility of the pHEA-SEC method to analyze antibodies without additional method devel-
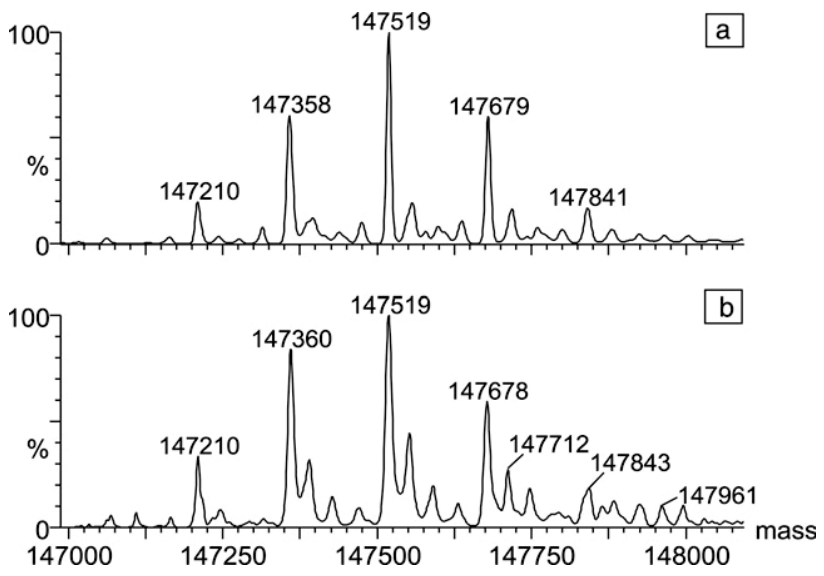

Figure 3. Comparison of the deconvoluted data obtained from pHEA-SEC (a) and rpHPLC (b). Signals have been normalized to the most intense species in each trace. The mass data has been reported to the nearest Dalton to be consistent with the 1.0 Da resolution used by MaxEnt1 to deconvolute these data. The mass accuracy for the major species in each sample was $24 \mathrm{ppm}$. opment, we tested several different antibodies under non-reducing conditions. The observed mass values and mass errors for these samples are reported in Table 1. Antibody ' $\mathrm{A}$ ' listed in this table corresponds to the data presented in Figures 1-3. These values, all below $25 \mathrm{ppm}$ in mass accuracy, show that this method is broadly applicable to the intact mass analysis of antibodies.

Another advantage of pHEA-SEC is the ability to efficiently desalt proteins of vastly different sizes. To demonstrate this aspect of the separation, we applied pHEA-SEC to resolution of reduced light and heavy chains corresponding to Antibody ' $\mathrm{A}$ '. Reduced antibodies provide a good test of the method since reduction typically uses high concentrations of denaturants, reducing agent, and other salts, in contrast to the analysis of intact antibodies. The UV trace shown in Figure 4 shows the separation of antibody light and heavy chains in the presence of $6 \mathrm{M} \mathrm{GnHCl}$ and $20 \mathrm{mM}$

Table 1. Observed mass values for non-reduced antibodies by pHEA-SEC MS*

\begin{tabular}{ccc}
\hline Antibody & $\begin{array}{c}\text { Major observed } \\
\text { mass }(\mathrm{Da})\end{array}$ & $\begin{array}{c}\text { Agreement with } \\
\text { expected (ppm) }\end{array}$ \\
\hline \hline Antibody A & $147,519 \mathrm{Da}$ & 24 \\
Antibody B & $149,384 \mathrm{Da}$ & 5.4 \\
Antibody C & $148,247 \mathrm{Da}$ & 22 \\
Antibody D & $147,098 \mathrm{Da}$ & 6.1 \\
Antibody F & $147,190 \mathrm{Da}$ & 7.1 \\
\hline
\end{tabular}

* Intact mass data obtained for five antibodies by pHEA-SEC MS. Spectra were summed and mass values determined as discussed in the Experimental section. 

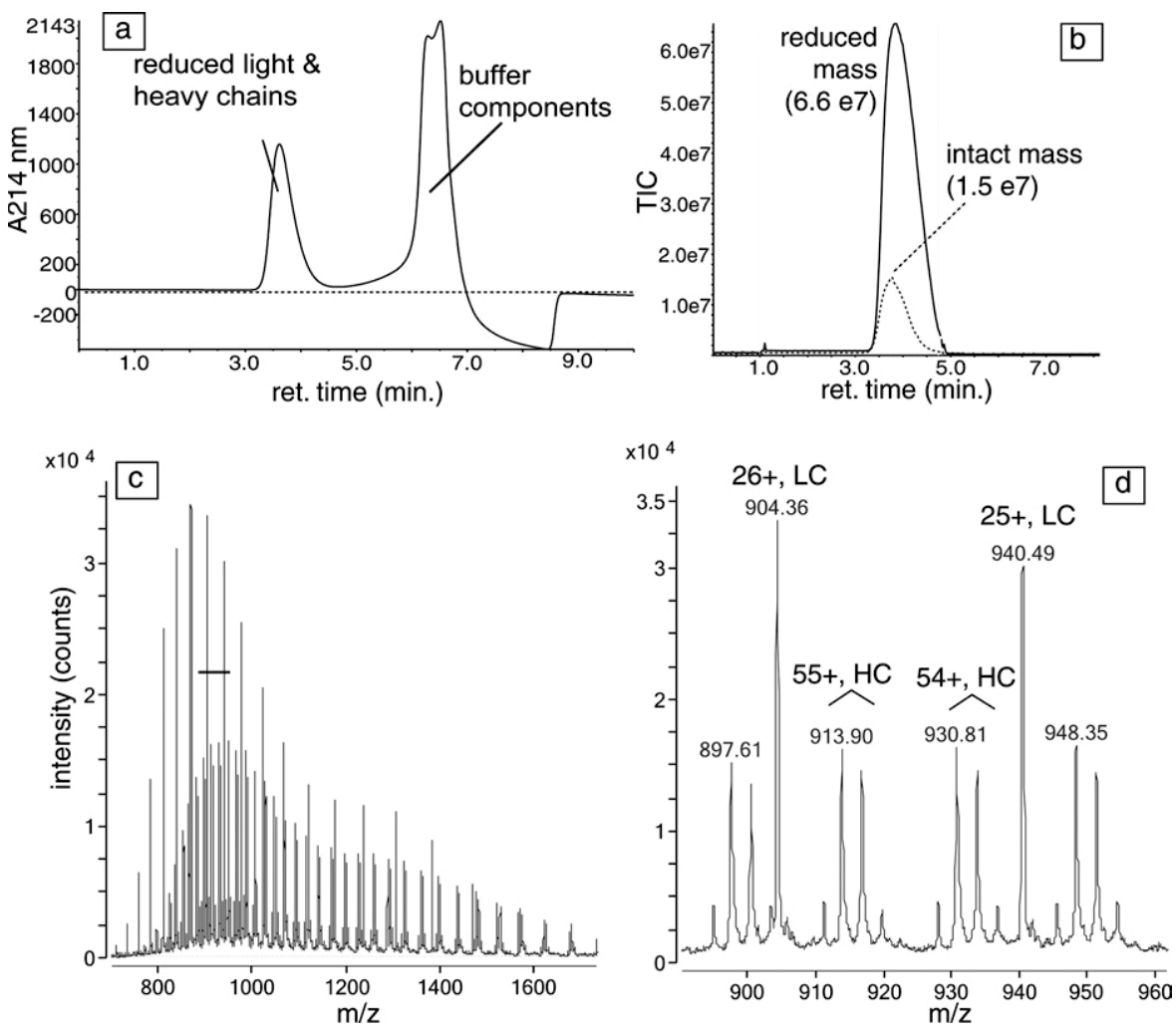

Figure 4. $\mathrm{A}_{214} \mathrm{~nm}$ UV data (a) and raw ESI-MS (c) spectra for reduced antibody 'A.' (b) Shows a comparison of the TIC traces for the reduced method and the intact mass data (4 $\mu \mathrm{g}$ load level) from Figure 1. (d) Shows a magnified region of the spectra shown in (c) from the region indicated by the solid bar superimposed on the image.

DTT. In contrast to the nearly perfectly Gaussian peak observed for intact antibody (see Figure $1 b$ ), a peak eluting in the same location, but with a somewhat less ideal peak shape, was observed. Additionally, a second, more intense peak corresponding to the salts and DTT present in the sample was observed to elute just after 6 min. As for intact mass analysis, a negative deflection of the baseline due to a local decrease in formic acid concentration was observed at $\sim 7.5 \mathrm{~min}$, marking the total volume of the column. A comparison of the TIC trace with that from the previously discussed nonreduced data showed an increase in signal (with equivalent sample loads) of about 6-fold for the reduced sample. This is consistent with the relatively stronger ionization of reduced light and heavy chains compared with intact antibody. Analysis of the spectra corresponding to the reduced protein peak indicated that heavy chain was slightly biased to the earlier eluting region of the peak but separation between the two was insufficient to allow separate deconvolution. For further analysis, the entire protein elution area was integrated to obtain a mixed spectrum with both light and heavy chain components as shown in the lower panel of Figure 4.

Comparison of the ionization profiles for the two components of the sample showed roughly 2-fold higher signal intensity for light chain, but close inspec- tion of the data showed good ionization for heavy chain and allowed multiple ions present at each charge-state to be distinguished. When the greater mass and heterogeneity of heavy chain (due to glycosylation) is taken into account, the lower signal relative to light chain is explained. Deconvoluted traces corresponding to reduced antibody 'A' are shown in Figure 5. Comparison of the observed and expected masses for each subunit showed mass accuracy for each was below $25 \mathrm{ppm}$. For heavy chain, heterogeneity due to glycosylation was resolved, primarily due to terminal galactosylation differences as discussed for intact mass data above.

To demonstrate the potential for the method as a screening tool, we tested a reduced antibody sample derived from clarified cell-culture supernatant. Deconvoluted data corresponding to the heavy chain subunit for the control and test sample is shown in lower panels of Figure 5. The high-quality of the data allowed differences in the glycosylation profile to be observed. This type of analysis shows the ability of this method to be used for samples from a challenging sample matrix where protein concentrations may be low in comparison to levels of interfering components that are often poorly removed by rpHPLC. This example illustrates the potential of this method for analysis of in-process samples.

Finally, we analyzed a sample of antibody (antibody ' $\mathrm{D}$ ' in Table 1) enriched for dimeric species as deter- 

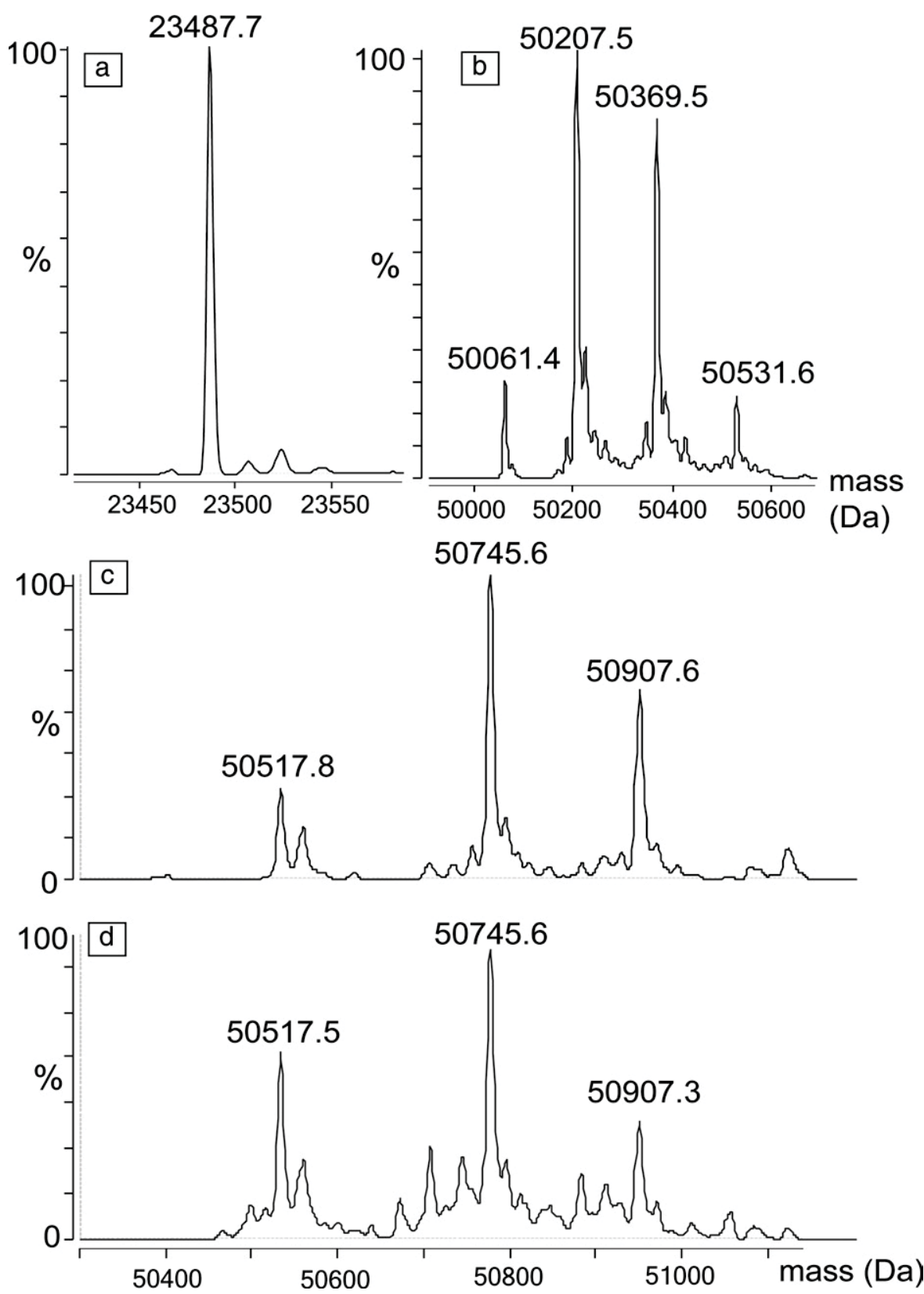

Figure 5. Comparison of the deconvoluted data for two reduced antibodies. Reduced data corresponding to antibody ' $\mathrm{A}$ ' is shown for light chain in (a) and heavy chain in (b). Each deconvoluted spectrum has been normalized to $100 \%$ for each displayed region. Mass accuracy for light chain was $21.2 \mathrm{ppm}$ and $0.2 \mathrm{ppm}$ for heavy chain (major species). Comparison of mass accuracy values for light and heavy chains from several separately prepared samples (analyzed on different days) showed typical mass accuracy ranges of 5 to $25 \mathrm{ppm}$ for each chain. A comparison of deconvoluted data for the heavy chain of an antibody analyzed directly from a cell-culture supernatant (d) is compared with control (c), with mass accuracy of $9 \mathrm{ppm}$ for the major species shown in each. It should be noted that minor species present in the deconvolution of reduced samples are not typically artifacts (as noted in the text for intact antibodies) but can usually be related to components in the sample by orthogonal methods.

mined by routine SEC analysis (details about the sample, but not mass analysis data, have been previously published [16]). Analysis of aggregated antibody samples by mass spectrometry is a challenge, made even more so by the difficulty of obtaining efficient desalting before sample introduction to the mass spectrometer.
Separation of aggregates by rpHPLC usually results in extremely poor peak shapes, inefficient desalting, and difficulty in fully eluting the protein from the hydrophobic matrix. To reduce sample heterogeneity and simplify interpretation of the mass data, samples were treated before analysis with PNGaseF to remove 
N-linked carbohydrates. Separation of the dimeric sample by pHEA-SEC resulted in a similar UV chromatogram to that shown for intact antibody in Figure 1. Figure 6 shows a comparison of the raw and deconvoluted data for control and dimeric samples analyzed under non-reducing conditions.

Comparison of the raw spectra for each sample showed the presence of a single charge-state envelope for the control (monomeric) sample and two overlapping envelopes for the dimeric sample. An overlay of the two multiply charged spectra showed that every other species was unique to the dimeric sample, suggesting the presence of both monomer and dimer. To compare the data, each sample was deconvoluted to include both monomeric and dimeric mass output ranges. Due to the larger peak width of the data for the dimer sample, a peak width value of $2.5 \mathrm{Da}$ was used for the deconvolution program; this setting was also used for the monomeric sample to provide comparative data. Narrower peak widths could not be used for the dimer sample without anomalous deconvolution results (data not shown). Use of a narrower peak width (see Figure 6) for the control sample resulted in a more accurate monomer mass nearly 20 Da lower in mass and closer to the expected value. The use of the wider peak width for both samples was done to demonstrate that no appreciable difference in monomer mass value was determined by deconvolution. Comparison of the deconvoluted data for each sample showed the presence of a $144 \mathrm{kDa}$ monomeric species of nearly identical mass. For the dimeric sample only, an additional species was observed at $288 \mathrm{kDa}$ consistent with a covalent dimer. This observation was supported by additional data beyond the scope of this report that showed the a

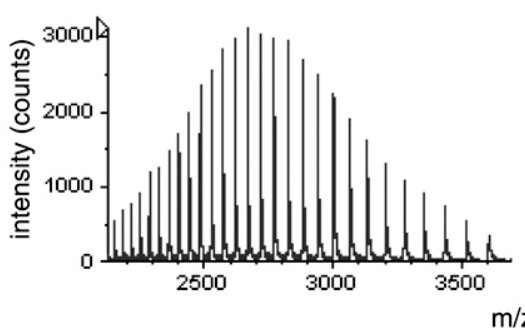

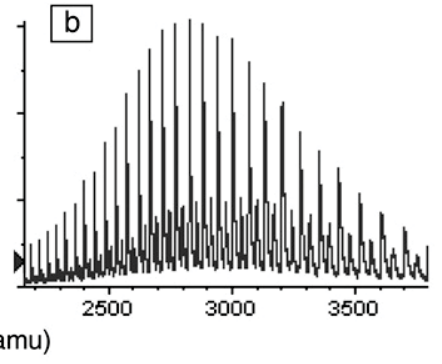

d
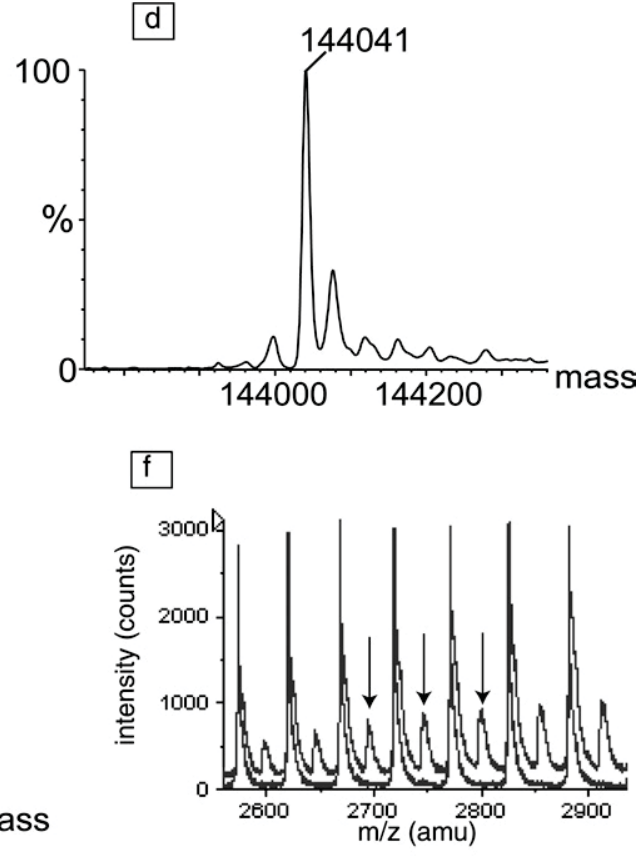

Figure 6. Raw and deconvoluted mass results for an antibody dimer compared with control. Raw spectra for the monomeric control (a) show a single multiply charged spectrum, while those corresponding to dimer (b) show two overlapping charge-state series. Deconvolution of both raw spectra (using a 2.5 Da peak width) across the range of monomer and dimer output mass values showed only monomeric mass for the control (c) but the presence of both monomer and dimer masses for the dimer sample (e). (d) Shows an example of the control sample deconvoluted with a $0.75 \mathrm{Da}$ peak width with an expanded view of the monomer mass. Mass accuracy for the 0.75 Da peak width analysis of the control was 23 ppm; mass accuracy for the control analyzed with a 2.5 Da peak width was $137 \mathrm{ppm}$. (f) Shows an overlay of the spectra for the monomer control and the dimer sample; arrows indicate charge states that are specific to the dimeric species only. 
presence of both covalent and noncovalent dimeric species within the sample.

The successful analysis and deconvolution of a dimeric antibody of nearly $300 \mathrm{kDa}$ in mass shows the flexibility of this simple but powerful sample introduction procedure. Combined with the results from reduced antibody analysis, this example shows that this method can be applied to proteins over 10-fold size difference (23.4 kDa light chain to $288 \mathrm{kDa}$ antibody dimer) without additional method development.

\section{Conclusions}

We report a method optimized on a widely available column that allows easy analysis of antibodies under either non-reduced or reduced conditions by mass spectrometry. This fast method has an analysis time below 10 min per sample and shows greater ionization sensitivity when coupled with ESI-MS than similar analyses achieved by rpHPLC. Additionally, the ability to operate the column at ambient temperature is advantageous, and minimizes the potential for analysis artifacts due to chromatographic column heating. Experiments performed with reduced, in-process, intact, and dimeric antibody samples show the flexibility of the method for different sample types. In addition to the data presented here, we have successfully used this method to characterize fractions from analytical scale cation exchange and hydrophobic interaction chromatography. This method should prove powerful wherever mass data is required to characterize samples purified by an orthogonal method, especially where those methods result in samples in high concentrations of salts or other buffer components.

\section{Acknowledgments}

We gratefully acknowledge the contributions of Sibylle Wilbert of ZymoGenetics, Inc. to the development of this method. We thank our Amgen colleague, Zhongqi Zhang, for helpful comments on the manuscript and Dean Pettit for supporting this work.

\section{References}

1. Dillon, T.; Bondarenko, P. V.; Ricci, M. S.; Development of an Analytical Reversed-Phase High-Performance Liquid Chromatography-Electrospray Ionization Mass Spectrometry Method for Characterization of Recombinant Antibodies. J. Chromatogr. A 2004, 1053, 299-305.

2. Chelius, D.; Jing, K.; Lueras, A.; Rehder, D. S.; Dillon, T. M.; Vizel, A.; Rajan, R. S.; Li, T.; Treuheit, M. J.; Bondarenko, P. V. Formation of Pyroglutamic Acid from N-Terminal Glutamic Acid in Immunoglobulin $\gamma$ Antibodies. Anal. Chem. 2006, 78, 2370-2376.

3. Yan, B.; Valliere-Douglass, J.; Brady, L. J.; Steen, S.; Han, M.; Pace, D.; Elliott, S.; Yates, Z.; Han, Y.; Balland, A.; Wang, W.; Pettit, D. Analysis of Post-Translational Modifications in Recombinant Monoclonal Antibody IgG1 by Reversed Phase Liquid Chromatography/Mass Spectrometry. I. Chromatogr. 2007, 1164, 153-161.

4. Dillon, T.; Bondarenko, P. V.; Rehder, D. S.; Pipes, G. D.; Kleeman, G. R.; Ricci, M. S. Optimization of a Reversed-Phase High-Performance Liquid Chromatography/Mass Spectrometry Method for Characterizing Recombinant Antibody Heterogeneity and Stability. J. Chromatogr. A 2006, $1120,112-120$.

5. Yang, J.; Wang, S.; Lui, J.; Raghani, A. Determination of Tryptophan Oxidation of Monoclonal Antibody by Reversed Phase High Performance Liquid Chromatography. J. Chromatogr. A 2007, 1156, 174-182.

6. Chu, G.; Chelius, D.; Xiao, G.; Khor, H.; Coulibaly, S.; Bondarenko, P. Accumulation of Succinimide in a Recombinant Monoclonal Antibody in Mildly Acidic Buffers Under Elevated Temperatures. Pharm. Res. 2007, 12, 1145-1156.

7. Brady, L. J.; Martinez, T.; Balland, A. Characterization of Nonenzymatic Glycation on a Monoclonal Antibody. Anal. Chem., in press.

8. Cordoba, A. J.; Shyonga, B.-J.; Breen, D.; Harris, R. J. Nonenzymatic Hinge Region Fragmentation of Antibodies in Solution. J. Chromatogr. B 2005, 818, 115-121.

9. Tous, G. I.; Wei, Z.; Feng, J.; Bilbulian, S.; Bowen, S.; Smith, J.; Strouse, R.; McGeehan, P.; Casas-Finet, J.; Schenerman, M. A. Characterization of a Novel Modification to Monoclonal Antibodies: Thioether Cross-Link of Heavy and Light Chains. Anal. Chem. 2005, 77, 2675-2682.

10. Mirza, U. A.; Chait, B. Effects of Anions on the Positive Ion Electrospray Ionization Mass Spectra of Peptides and Proteins. Anal. Chem. 1994, 66 $2898-2904$.

11. Wan, H. Z.; Kaneshiro, S.; Frenz, J.; Cacia, J. Rapid Method for Monitoring Galactosylation Levels During Recombinant Antibody Production by Electrospray Mass Spectrometry with Selective Ion Monitoring. J. Chromatogr. A 2004, 1053, 299-305.

12. Alpert, A. J. Hydrophilic Interaction for the Separation of Peptides, Nucleic Acids, and Other Polar Compounds. J. Chromatogr. A 1990, 4999, 177-186

13. Shen, M. L.; Benson, L. M.; Johnson, K. L.; Lipsky, J. J.; Naylor, S. N. Effect of Enzyme Inhibitors on Protein Quaternary Structure Determined by On-Line Size Exclusion Chromatography-Microelectrospray Ionization Mass Spectrometry. J. Am. Soc. Mass Spectrom. 2001, 12 97-104.

14. Lazar, A. C.; Wang, L.; Blätter, W. A.; Amphlett, G.; Lambert, J. M.; Wang, W. Analysis of the Composition of Immunoconjugates Using Size-Exclusion Chromatography Coupled to Mass Spectrometry. Rapid Commun. Mass Spectrom. 2005, 19, 1806-1814.

15. Gadgil, H.; Pipes, G.; Dillon, T.; Treuheit, M.; Bondarenko, P. Improving Mass Accuracy of High Performance Liquid Chromatography/Electrospray Ionization Time-of-Flight Mass Spectrometry of Intact Antibodies. J. Am. Soc. Mass Spectrom. 2006, 17, 867-872.

16. Michels D. A.; Brady L. J.; Guo A.; Balland A. Fluorescent Derivatization Methods of Proteins for Characterization by Capillary ElectrophoresisSodium Dodecyl Sulfate with Laser-Induced Fluorescent Detection. Anal. Chem. 2007, 79, 5963-5971. 